\title{
SAFETY TRAINING FOR SCAFFOLDING AND FORMWORK CONSTRUCTION BY USING VIRTUAL ENVIRONMENT
}

\author{
Isik Ates Kiral ${ }^{1}$ and Semra Comu ${ }^{2}$
}

\begin{abstract}
Scaffolding and formwork activities are the most frequent construction activities that exist in almost every construction project. Common site accidents such as falling from a height, formwork collapses frequently occur during these activities. Previous literature highlight that human-based factors are the major causes that lead to the scaffolding and formwork accidents. In this sense, safety training is crucial to prevent these accidents by improving the safety skills of trainees. Knowing that virtual technologies based trainings have a great potential to provide an effective training, we developed a virtual safety training tool called V-SAFE.v2 which simulates the scaffolding and formwork activities. V-SAFE.v2 supports both single and multi-user training scenarios. In the training stage, trainees interact with the autonomous agents to learn the essentials of the tool and the scaffolding and formwork construction skills. In the testing stage, trainees enter into the virtual environment to test their skills and safety knowledge that acquired during the training stage. Finally, trainees receive feedback based on their safety behavior at the end of testing stage. The preliminary results of this study demonstrate that VSAFE.v2 is an effective safety training tool for the scaffolding and formwork activities.
\end{abstract}

Keywords: Scaffolding and Formwork, Virtual Construction Safety Training, Safety Management, Virtual Environment

\section{INTRODUCTION}

The number of the occupational fatal accidents highlight that construction is one of the most high-risk industries around the world. Fatal accidents in the US construction industry increased by 4 percent in 2015 to 937 (Bureau of Labor Statistics (BLS) 2016a). Comparing to other industries, construction industry had the highest number of the occupational fatalities in 2015; the highest total since 975 fatal cases in 2008. (BLS 2016a). Quite similarly, 217 fatal accidents were recorded between 2010 and 2015 in the British construction industry (Health and Safety Executive (HSE) 2016). Likewise, around 3,739 fatal injuries recorded in 28 European countries in 2014, and 20,9\% of all fatal accidents occurred in the construction industry (Eurostat 2016). Accordingly, the construction industry has the highest fatal accident rate in the 28 European Union countries. Therefore, the high number of accidents and fatalities in the global construction industry indicates that effective safety management should be provided to mitigate and prevent the hazards on construction sites.

According to Occupational Safety and Health Administration (OSHA) (2016), 65\% of the site employee work on scaffolds since it is one of the most frequently used construction

1 PhD Candidate, Department of Civil Engineering, Bogazici University, Bebek 34342 Istanbul, Turkey, ates.kiral@boun.edu.tr

2 Associate Professor, Department of Civil Engineering, Bogazici University, Bebek 34342 Istanbul, Turkey, semra.comu@boun.edu.tr 
equipment in every construction project. Therefore, protecting workers from scaffoldrelated accidents may prevent some of the accidents on the construction sites. Scaffolding contributes several benefits during many construction activities. First of all, it provides field employees to work temporarily at a height. Moreover, scaffolding platform enables a safe workplace to the field employees. On the other hand, lack of knowledge or improper safety behavior may cause accidents such as the structure collapses or falling from a height during the scaffolding activities (Nadhim et al. 2016). According to the BLS, 79 scaffolding accidents were recorded in the United States, in 2015 (2016b). Similarly, among different construction activities, 97 falling from a height accidents involved scaffolding activities in the United Kingdom between 2010 and 2015 (HSE 2016). Accordingly, falling from a height corresponds to $8.4 \%$ of the all work-related deaths in the United States (BLS, 2016b) and $44.7 \%$ in the United Kingdom (HSE 2016). Likewise, formwork activities are also another common type of construction activities that leads to serious accidents on construction sites such as exposure to harmful substances, improper lifting, falling from height and striking by an object or vehicle, etc. (Hallowell and Gambatese 2009; Jurewicz 1988). Previous literature (Hallowell and Gambatese 2009; Jurewicz 1988) highlight that improper lifting, lack of precaution during the design and poor risk classification are the major causes of the accidents during the formwork activities.

Therefore, effective safety training is crucial to prevent the accidents occurred during scaffolding and formwork activities. In this case, virtual environments have a great potential to provide an effective and highly engaging safety training. In this study, we established a virtual environment based training procedure to improve the safety training for scaffolding and formwork construction.

\section{RESEARCH BACKGROUND}

Many studies (e.g. Nadhim et al. 2016; Sawacha et al. 2014; Halperin and McCann 2004, Whyte and Brandis 2012) highlight that human-based factors are the major causes of the accidents during scaffolding and formwork activities. Nadhim and colleagues (2016) conducted a meta-analysis to describe the characteristics of the falling from height accidents, which is quite common during the scaffolding and formwork activities. Study results show that $23 \%$ of the falling from height accidents occurred during the scaffolding activities (Nadhim et al. 2016). Moreover, the same study also highlights that individual characteristics of the field workers such as knowledge level, lack of education, experience, training, and safety behavior are the second most important causes of the falling from height accidents, while the most important cause is the risky characteristics of falling from height activities such as the complexity of the activity, hardship and prolong tasks (Nadhim et al. 2016). Similarly, Sawacha and colleagues (1998) identified the factors affecting the site safety. Results show that scaffolding is one of the major risk sources on the construction field (Sawacha et al. 1998). Moreover, Sawacha and colleagues (1998) also propose that technical skills, efficient training and work experience are crucial to prevent scaffolding accidents. On the same vein, Halperin and McCann (2004) analyzed the effectiveness of the scaffolding practices from the safety point of view. They (Halperin and McCann 2004) claim that using the safer inspection method provides on-site safety during the scaffolding tasks. Therefore, effective safety training should be provided to improve the safety skills of the field employee. In other words, providing effective safety training helps to ensure a safe workplace during scaffolding and formwork activities which may mitigate or prevent fatal accidents related to scaffolding activities. 
In order to provide an effective safety training, previous studies (Kirkpatrick 1979; Burke et al. 2006) commonly acknowledge the importance of hands-on training on the construction site. Firstly, Kirkpatrick (1979) developed a safety training effectiveness model based on the behavior and knowledge level of the trainees. Kirkpatrick (1979) argue that without hands-on practice, providing an effective safety method is not possible. Similarly, Burke and colleagues (2006) developed a safety training effectiveness framework. Burke and colleagues (2006) argue that an effective safety training method should involve information transfer, hands-on training, behavioral modelling, and feedback mechanism. In other words, current literature highlight that a robust safety training method should enable the conveyance of safety information via hands-on practice. On the other hand, providing an effective safety training is a challenging task in the construction industry, since, hands-on practice on the construction site is quite costly and even risky. Therefore, construction companies prefer using conventional methods such as lectures, videos, etc. which fails to adopt hands-on practice.

In this case, virtual environments have a great potential to fulfill the need of hands-on practices in the construction industry. Virtual environment establishes a base for the users to evaluate the social situations and the outcome of their decisions in a risk-free environment. Thus, users could accomplish their learning goals by interacting with a realistic work environment. Embedding the scaffolding and formwork activities into a virtual environment could support the learning process of the participants in working both individual, and collaborative tasks. Recently, many studies (Lin et al. 2011; Son et al. 2011; Albert et al. 2014; Kural et al. 2016) focus on the virtual construction safety training area. Lin and colleagues (2011) integrated a simulation-based tower crane virtual safety training education. Study results show that instructional-based virtual education improved the performance of the trainees on the tower crane activities. Quite similarly, Son and colleagues (2011) involved a virtual environment based safety training and study results show that the visualization feature of virtual reality improves the hazard identification level of the users. On the same vein, Albert and colleagues (2011) defined energy sources on the virtual environment, in order to enhance hazard identification level of the participants. They point out that the memory capacity of the users is significantly improved via virtual practice, thus, their hazard identification level increased (Albert et al., 2011). Moreover, Kiral and colleagues (2016) developed a virtual safety training tool for tower crane operations entitled V-SAFE (Virtual Safety Analysis For Engineering applications). Accordingly, they specify the features required for effective safety training by using virtual environments (Kiral et al. 2016).

Consequently, many studies in the literature highlight the advantages of utilizing the virtual environments in the construction safety training. On the other hand, there are a limited number of studies that concentrate on the scaffolding and formwork activities. In the lights of these issues, we aim to develop the second version of the virtual safety training tool called V-SAFE based on the scaffolding and formwork activities. In this study, we introduce and discuss the important features of V-SAFE.v2 in terms of providing effective safety training including hands-on practices for scaffolding and formwork activities.

\section{RESEARCH METHODOLOGY AND DisCUSSION}

In this study, we aim to develop a virtual environment based safety training tool for scaffolding and formwork activities. V-SAFE.v2 simulates the major tasks of the scaffolding and formwork activities in a 3D virtual environment. The main characteristics of V-SAFE.v2 are the surrounding environment, reality integration, collision detection and 
scenario development, which improve the simulation level of the activities. Thus, trainees could improve their safety skills such as improving hazard identification level, hands-on practice experience, safety behavior patterns, etc.

\subsection{V-SAFE.v2 Tool Development}

In the first version of the software (V-SAFE.v1), a tower crane operation was simulated in a 3D virtual media (i.e. Kiral et al. 2015, Kiral et al. 2016). In the following version of the tool (V-SAFE.v2), scaffolding and formwork activities that are the most frequent construction activities in many construction project are simulated. V-SAFE.v2 is developed by using the Unity game engine. Unity is firstly designed to develop video games for PCs, gaming consoles, mobile devices, and websites. Yet, it has not only been used for gaming purposes, but also for architecture, engineering and construction (AEC) services. In order to meet the visualization requirement of the engineering applications, Unity is based on an intuitive and flexible nature. Arch Virtual, Zahner and NVYVE are some of the licensees of Unity from the AEC industry (Unity 2016). Moreover, to meet the engineering specifications, Unity has partnered with VIMTREK which provides BIM solutions and 3D visualization to the owners, designers and contractors. VIMTREK takes the BIM models and turn them into 3D, interactive, virtual environments by using the Unity's engine.

Another major component of V-SAFE.v2 is the cloud-based learning management system (Cloud LMS). Learning management system is a novel e-learning technology, which "is a software application for the administration, documentation, tracking, reporting and delivery of electronic educational technology (also called e-learning) courses or training programs" (Devisri 2016; p.77). By using the cloud management system, we aim to store and analyze the necessary information in V-SAFE.v2.

In V-SAFE.v2, trainees initially create their account on the cloud based LMS and select their role and avatar. After the selection of their avatars and simulation role, Unity starts the scenario and users could begin to interact with each other. During the virtual interaction with other users and media, trainees use keyboard and mouse layout. The users' viewpoint is based on the third person view and they could interact with each other and virtual objects via point and click structure. Accordingly, users could click on the necessary construction object (Figure 1) to fulfil the requirements of their role in the virtual scaffolding and formwork activity.

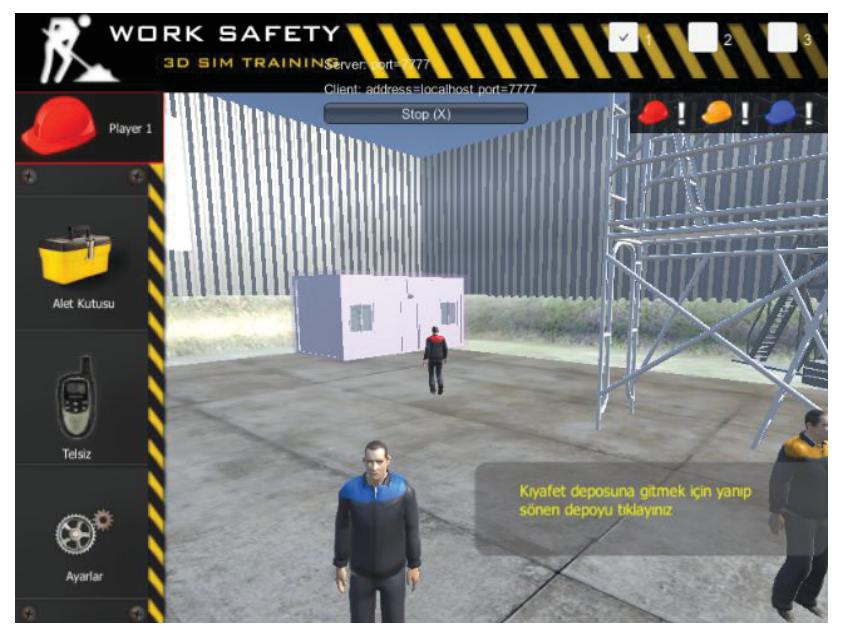

Figure 1: A view of V-SAFE.v2 


\subsection{V-SAFE Training Framework}

The development framework of the V-SAFE involves five major stages presented as follows:

Stage 1: Information about the scaffolding and formwork safety, such as the precautions, hazards, precursors lead to the hazards are gathered from the safety regulations, the accident reports and the previous studies in the literature. Accordingly, based on the retrieved information, the training scenario is developed.

Stage 2: Hazard information, precursors that lead to the hazards and the safety prevention methods, which gathered in the first stage, are stored in the Cloud LMS. Later on, by analyzing the probability, impact and prevention methods of the hazards, the safety training procedure is developed and integrated into the scenario.

Stage 3: Based on the safety training procedure established in the second stage, a pilot learning module (Module 2a) is developed. In module 2a, only a single trainee can run the simulation. Initially, the trainee selects his/her role in a single player platform. For this reason, Module 2a consists of autonomous agents. During the simulation, autonomous agents act with correct safety behavior. In other words, autonomous agents behave in correct safety attitudes, with full safety knowledge in the learning stage of the tool. Moreover, the trainee is guided through written assistance and flashing objects by the software. In module 2a, users need to follow the instructions, thus, learn the basics of the software and safety precautions of the scaffolding and formwork activities. After completing the instructions from the software, trainee finishes the Module $2 \mathrm{a}$ - Pilot Learning Module.

Stage 4: Once the learning module is successfully completed by the trainees, they may proceed with the following testing module. The scenario of the Module $2 \mathrm{~b}$ is similar to the Module 2a, but, the main difference from the Module 2a is the multiplayer function without having autonomous agents. In the Module $2 \mathrm{~b}$, three users need to fulfill their assignments without any guidance from the software. Based on their safety knowledge level, users have been assessed in Module 2b automatically. Another major difference could be considered as more concentration on the behavior modeling and hands-on practice comparing to Module 2a.

Stage 5: Module 2c is again based on the incidence scenario of the scaffolding and formwork accidents. This module is the second testing version for a single user with autonomous agents. This module may integrate the autonomous agents with wrong safety behavior. With this way, trainees can observe the results of wrong safety attitudes and actions during construction tasks. Also, the integrated autonomous agents can act in correct safety behavior, in that case the performance of a single trainee can be evaluated.

\subsection{Sense of Reality}

Previous literature (Albert et al. 2014) highlight that the sense of reality is an important parameter in virtual training procedure. For this reason, the role of the game engine and the way virtual reality technologies is utilized are quite important. The fundamental reason of using the virtual reality is enabling visual human-machine interaction by the provision of the visual-based information flow in the virtual environment (Hoffman et al. 1996). In that case, rendering the physical real objects is completed via using the virtual environment. In the virtual safety training, the sense of reality is developed through improving the spatial awareness of the trainees. By improving the quality of the visualization, the trainees could enhance their comprehensibility level. In that sense, using Unity is advantageous due to using its superior models for construction projects. Also, 
Cloud LMS provides a structured learning with the usage of the probability and impact analysis of risk sources for the autonomous agents

\subsection{Autonomous Agent Support, Instructional Feedback, Learning Types and Behavior Modelling}

One of the major features of V-SAFE.v2 is the integration of autonomous agents. Previous literature (Burke et al., 2006) highlights the importance of behavior modelling in the safety training. By using autonomous agents, a controlled environment could be set up in both training module and testing module. In the pilot training part (Module 2a), trainees can learn the specifics of their task and gain safety knowledge from autonomous agents by observing correct work skills and safety behavior. Moreover, in the testing stage autonomous agents with erroneous skills and safety behaviors are also utilized, with this way trainees are given the opportunity of observing the consequences of wrong actions on the site. Integrating autonomous agents into the virtual environment is a new approach in the construction safety training literature. With this novel approach, it is possible to improve the safety performance of trainees.

Moreover, V-SAFE.v2 provides instructional feedback for each trainee based on their performance. Burke and colleagues (2006) state that an instructional feedback should be specific and based on each users' weaknesses and safety knowledge level. Moreover, they (Burke et al. 2006) also claim that least engaging methods fail to provide behavioral modeling. In the lights of this context, V-SAFE.v2 provides an individual and instructional feedback based on the trainees' safety performance. At the end of the testing module, trainees receive a detailed list of their mistakes. During evaluation, behavior-based automated assessment system is used. This method is also a new approach since, previous studies opt for using conventional evaluation methods to understand the weaknesses of the trainees. In this context, we are developing a feedback procedure based on the safety behavior of trainees, rather than their knowledge level. Thus, trainees learn from their mistakes and improve their knowledge level and safety behaviors.

Another important feature of V-SAFE.v2 is the establishment of a base for both, individual and interpersonal learning. In the learning process, Brown et al. (1989) emphasize the importance of self-learning especially for the trainees with the insufficient knowledge. For the safety training context, self-learning is very crucial, since the workers' safety knowledge level could be low. Various reports (BLS 2016a; HSE 2016) show that accidents occurred during scaffolding activities such as striking by fallen objects and falling from a height are quite repetitive. In that case, the main reason of the accidents might be considered as the inadequacy of conventional safety training methods in terms of transferring the safety information and enabling knowledge development. In that case, the visual quality of virtual media is supportive to improve the comprehensibility of the safety information by the trainees. Nadhim et al. (2016) also indicate that the main causes of scaffolding related accidents are lack of safety information and getting poor training. In this sense, the training module of V-SAFE.v2 contributes to the learning of both scaffolding activity skills and related correct safety actions.

Just like self-learning, interpersonal learning is another important type of the learning methodology (Earle 1971). Many scaffolding and formwork activities require collaboration such as locking the support to the column formwork, installing the column formwork, etc. To enhance the collaboration skills of trainees, V-SAFE.v2 provides many collaborative tasks in the virtual environment, in other words V-SAFE.v2 is a multi-user tool which enables the collaboration between participants and interaction with the virtual objects. Accordingly during the testing part, users could also learn from each other through 
collaboration. Therefore, V-SAFE.v2 has a high potential to enable two learning models; individual and interpersonal learning.

Another important feature of V-SAFE.v2 is improving the behavior-based skill sets. As mentioned, several accidents such as falling from height, struck by falling objects occur very frequently during the scaffolding activities. The precautions for these frequent accident types are actually not complicated. By only wearing full-body harness, preventing the falling from height accidents on the scaffolding activities is possible. In that case, the major reason of falling from height is not the lack of safety knowledge, the main reason is the improper safety attitudes. By realizing the consequence of a catastrophic event in the virtual environment, the users might adjust their safety attitudes on the real construction site.

In this study, several limitations exist. First of all, since the test module of V-SAFE.v2 is still under development, representing empirical results is not possible at this stage. Consequently, we merely concentrated on describing the major features of V-SAFE.v2 and identifying the training and testing framework. In the following stages of the research, we plan to conduct several experiments to test the effectiveness of V-SAFE.

\section{CONCLUSIONS}

Scaffolding and formwork tasks are one of the complex activities in the construction industry. Due to this complexity, providing safety on these tasks is quite challenging. Previous literature highlighted the importance of the active, hands on practice during the safety training. Despite its importance, construction companies still prefer using the conventional safety training methods such as lectures, videos that fail to provide active, hands on training. In this study, we aimed to fill this gap by describing how V-SAFE.v2 could provide active, hands-on practice for scaffolding and formwork tasks without being exposed to the risks of real construction site. Therefore we define the main features of $\mathrm{V}$ SAFE v.2 and analyzed the potential benefits of the tool, in order to improve the safety performance level of trainees. To summarize, this study provides insights for the further studies that aim to develop a highly engaging safety training procedure.

\section{ACKNOWLEDGMENTS}

This work is supported by The Scientific and Technological Research Council of Turkey through Grant No. 315M186.

\section{REFERENCES}

Albert, A., Hallowell, M.R., Kleiner, B., Chen, A. and Golparvar-Fard, M. (2014). Enhancing construction hazard recognition with high-fidelity augmented virtuality. $J$. of Cons. Eng. and Managt., 140 (7), pp. doi: 10.1061/(ASCE)CO.1943-7862.0000860

Brown, J. S., Collins, A. and Duguid, P. (1989). Situated cognition and the culture of learning. Educational researcher, 18(1), 32-42. doi: 10.3102/0013189X018001032

BLS. 2016a. Fatal occupational injuries counts and rates by selected industries, 2014-15.

Available at: https://www.bls.gov/news.release/cfoi.t04.htm (Accessed on 01 Dec 2016).

BLS. 2016b. Fatal occupational injuries by selected characteristics, 2003-2015. Available at: https://www.bls.gov/iif/oshwc/cfoi/all_worker.xlsx (Accessed on 01 Dec 2016). 
Burke, M. J., Sarpy, S. A., Smith-Crowe, K., Chan-Serafin, S., Salvador, R. and Islam, G. (2006). Relative effectiveness of worker safety and health training methods. American Journal of Public Health, 96(2), 315 - 324

Devisri, K. (2016). E-Education Management Systems. In: Laxmi book Publication 1st ed. Book title. Place: Ashok Yakkaldevi, chapter 4 page 77.

Earle, T. C. (1971). Task Learning, Interpersonal Learning and Cognitive Complexity Oregon Research Institute Eugene.

Eurostat. (2016). Fatal Accidents at work by NACE Rev. 2 activity. Available at: http://appsso.eurostat.ec.europa.eu/nui/show.do?dataset=hsw_n2_02\&lang=en

(Accessed on 01 Dec 2016).

Hallowell, M. and Gambatese, J. (2009). Activity-Based Safety Risk Quantification for Concrete Formwork Construction. Journal of Construction Engineering and Management. 10.1061/(ASCE)CO.1943-7862.0000071, 990-998.

Halperin, K. M. and McCann, M. (2004). An evaluation of scaffold safety at construction sites. Journal of Safety Research, 35(2), 141-150.

Health and Safety Executive, (2016) Health \& Safety in Constr. Sector in GB 2014/15. Available at: http://www.hse.gov.uk/statistics/industry/construction/construction.pdf (Accessed on 01 Dec 2016).

Hoffman, H., Groen, Rousseau J. S., Hollander A., Winn W., Wells M., and Furness T. (1996). Tactile Augmentation: Enhancing Presence in Virtual Reality with Tactile Feedback from Real Objects, Meeting of the American Psycho- logical Society, SF.

Jurewicz, R. A. (1988). Worker safety in formwork operations. Concrete Construction, 303-10.

Kirkpatrick, D. L. (1979). Techniques for evaluating training programs. Training and development journal.

Kiral, I. A., Comu, S. and Kavaklıglu (2015). Enhancing the Construction Safety Training by Using Virtual Safety Training Tool: V-SAFE. The CSCE Conference, University of British Columbia, Vancouver, Canada.

Kiral, I.A., Çomu, S., Chen, J. and Taylor, J.E. (2016). Virtual Collaborative Safety Training for the Crane Operations, 16th International ConVR, Hong Kong, 11-13 Dec 2016

Lin, K. Y., Son, J. W. and Rojas, E. M. (2011). A pilot study of a 3D game environment for construction safety education. Journal of Information Technology in Construction, 16(5), 69-83.

Nadhim, E. A., Hon, C., Xia, B., Stewart, I., and Fang, D. (2016). Falls from height in the construction industry: a critical review of the scientific literature. International journal of environmental research and public health, 13(7), 638.

OSHA. (2016). Safety Requirements for Scaffolding. Available at: https://www.osha.gov/SLTC/scaffolding/ (Last accessed on 01 December 2016).

Sawacha, E., Naoum, S. and Fong, D. (1999). Factors affecting safety performance on construction sites. International journal of project management, 17(5), 309-315.

Son, J., Lin, K. Y. and Rojas, E. M. (2011). Developing and testing a 3D video game for construction safety education. Computing in Civil Engineering, 2011, 867-74.

Unity. (2016). Available at: https://unity3d.com/ (Last accessed on 01 December 2016). 